\title{
ARTICLE OPEN \\ Pivotal trial of an autonomous AI-based diagnostic system for detection of diabetic retinopathy in primary care offices
}

\author{
Michael D. Abràmoff (iD ${ }^{1,2,3,4}$, Philip T. Lavin ${ }^{5}$, Michele Birch ${ }^{6}$, Nilay Shah ${ }^{7}$ and James C. Folk ${ }^{1,2,3}$
}

\begin{abstract}
Artificial Intelligence (AI) has long promised to increase healthcare affordability, quality and accessibility but FDA, until recently, had never authorized an autonomous Al diagnostic system. This pivotal trial of an Al system to detect diabetic retinopathy (DR) in people with diabetes enrolled 900 subjects, with no history of DR at primary care clinics, by comparing to Wisconsin Fundus Photograph Reading Center (FPRC) widefield stereoscopic photography and macular Optical Coherence Tomography (OCT), by FPRC certified photographers, and FPRC grading of Early Treatment Diabetic Retinopathy Study Severity Scale (ETDRS) and Diabetic Macular Edema (DME). More than mild DR (mtmDR) was defined as ETDRS level 35 or higher, and/or DME, in at least one eye. Al system operators underwent a standardized training protocol before study start. Median age was 59 years (range, 22-84 years); among participants, $47.5 \%$ of participants were male; $16.1 \%$ were Hispanic, $83.3 \%$ not Hispanic; $28.6 \%$ African American and $63.4 \%$ were not; 198 (23.8\%) had mtmDR. The Al system exceeded all pre-specified superiority endpoints at sensitivity of $87.2 \%(95 \% \mathrm{Cl}$, 81.8-91.2\%) (>85\%), specificity of $90.7 \%(95 \% \mathrm{Cl}, 88.3-92.7 \%)(>82.5 \%)$, and imageability rate of $96.1 \%(95 \% \mathrm{Cl}, 94.6-97.3 \%)$, demonstrating Al's ability to bring specialty-level diagnostics to primary care settings. Based on these results, FDA authorized the system for use by health care providers to detect more than mild DR and diabetic macular edema, making it, the first FDA authorized autonomous Al diagnostic system in any field of medicine, with the potential to help prevent vision loss in thousands of people with diabetes annually. ClinicalTrials.gov NCT02963441
\end{abstract}

npj Digital Medicine (2018)1:39; doi:10.1038/s41746-018-0040-6

\section{INTRODUCTION}

People with diabetes fear visual loss and blindness more than any other complication. ${ }^{1}$ Diabetic retinopathy (DR) is the primary cause of blindness and visual loss among working age men and women in the United States and causes more than 24,000 people to lose vision each year. ${ }^{2,3}$ Adherence to regular eye examinations is necessary to diagnose DR at an early stage, when it can be treated with the best prognosis, ${ }^{4,5}$ and have resulted in substantial reductions in visual loss and blindness. ${ }^{6}$ Despite this, less than $50 \%$ of patients with diabetes adhere to the recommended schedule of eye exams, ${ }^{7}$ and adherence has not increased over the last 15 years despite large-scale efforts to increase it. ${ }^{8}$ To increase adherence, retinal imaging in or close to primary care offices followed by remote evaluation using telemedicine has also been widely studied. ${ }^{9-11}$

Artificial intelligence (AI)-based algorithms to detect DR from retinal images have been examined in laboratory settings. ${ }^{12-15}$ Recent advances incorporate improved machine learning into these algorithms have led to higher diagnostic accuracy. ${ }^{16,17}$ However, in addition to high diagnostic accuracy, responsible and safe implementation in primary care requires autonomy (i.e., a use case that removes the requirement for review by human experts), instantaneous image quality feedback to the primary care based operator in order to reach a reliable disease level output in the vast majority of patients, a realtime clinical decision at the point of care, and consistent diagnostic accuracy across age, race and ethnicity. ${ }^{12,13,18,19}$ Studies comparing an Al system against an independent, high-quality gold standard that includes fundus imaging and Optical Coherence Tomography (OCT) imaging protocols have not previously been conducted; FDA has not previously authorized any such system.

The Wisconsin Fundus Photograph Reading Center (FPRC) has historically been the gold standard for trials that require grading of the severity of DR, including the Epidemiology of Diabetes Interventions and Complications/Diabetes Control and Complications Trial (EDIC/DCCT), Diabetic Retinopathy Clinical Research Network (DRCR.net) studies, as well as pivotal phase III studies. ${ }^{20,21}$ The FPRC has adopted the use of a widefield stereoscopic retinal imaging protocol (4W-D), that includes four stereoscopic pairs of digital images per eye, each pair covering $45-60^{\circ}$, equivalent to the area of the retina covered by the older, modified 7-field stereo film protocol. ${ }^{22,23}$ Traditionally, the presence of Diabetic Macular Edema (DME) was determined from the stereo fundus photos, but more recently, Optical Coherence Tomography (OCT) has become the reference modality for determining the presence or absence of center-involved DME. ${ }^{24}$ The 2017 revision of the American Academy of Ophthalmology's Preferred Practice Pattern recommends people with no or mild DR be followed annually, whereas those with more than mild DR, and/or DME (abbreviated to $\mathrm{mtmDR}$ ), are recommended to receive evaluation and consideration for treatment. ${ }^{25,26}$

\footnotetext{
${ }^{1}$ Department of Ophthalmology and Visual Sciences, University of lowa, lowa City, IA 52242, USA; ${ }^{2}$ Veterans Administration Medical Center, lowa City, IA 52242, USA; ${ }^{3}$ IDx LLC Coralville, IA 52241, USA; ${ }^{4}$ Institute for Vision Research, University of lowa, lowa City, IA 52242, USA; ${ }^{5}$ Boston Biostatistics Research Foundation, Inc., 3 Cahill Park Drive, Framingham, MA 01702, USA; ${ }^{6}$ Department of Family Medicine, Director of Academic Services, University of North Carolina School of Medicine, Charlotte, NC 28204, USA and ${ }^{7}$ The Emmes Corporation, 401 North Washington Street, Suite 700, Rockville, MD 20850, USA Correspondence: Michael D. Abràmoff (michael-abramoff@uiowa.edu)
}

Received: 28 May 2018 Revised: 6 July 2018 Accepted: 10 July 2018 Published online: 28 August 2018 
In this study, we evaluate the diagnostic performance of an autonomous Al system for the automated detection of DR and DME, termed mtmDR. Study subjects were people with diabetes, not previously known to have DR or DME, under an intent-toscreen (ITS) study design. Ten study sites were all in primary care offices, and the Al systems were operated by existing staff at those sites, using standardized training and operator materials to facilitate use of the system. The FPRC imaging on the other hand was conducted by FPRC certified expert photographers.

\section{RESULTS}

Study population

A total of 900 participants were enrolled at 10 sites, of which 892 participants completed all procedures. A subset of 819 of these participants could be fully analyzed, see Fig. 1, giving an analyzable fraction of $92 \%$ (95\% Cl, 90\%-93\%). Median age was 59 years (range, $22-84$ years); $47.5 \%$ of participants were male. For the entire group of participants $16.1 \%$ were Hispanic, $83.3 \%$ were not Hispanic, and $0.6 \%$ unknown. Also, $63.4 \%$ were white, $28.6 \%$ African American, and $1.6 \%$ Asian (Table 1). Finally $7.1 \%$ had type 1 diabetes, 92.9\% had type 2 diabetes. The 819 participants whose results could be fully evaluated and the 73 participants whose results could not be analyzed, differed significantly with respect to lens status, while mean age, ethnicity, race, and $\mathrm{HbA} 1 \mathrm{C}$ level were not significantly different (Table 1). The enrichment strategy led to 319 enriched participants; the 621 No/Mild DR participants included 218 participants from enrichment while the $198 \mathrm{mtmDR}$ participants included 101 participants from enrichment. In the subset of participants enrolled without enrichment, mean $\mathrm{HbA1C}$ \pm std was $8.1 \pm 1.6 \mathrm{mmol} / \mathrm{l}$, while for all participants overall mean $\mathrm{HbA} 1 \mathrm{C} \pm$ std was $9.4 \pm 2.3 \mathrm{mmol} / \mathrm{l}$. According to the FPRC, $11 /$ 819 subjects had "enlarged cup to disc ratio", and 26/819 subjects had "any drusen" and/or "any retinal pigment epithelium atrophy"-none of these subjects had increased retinal thickness on OCT.

A total of $198 \mathrm{mtmDR}$ participants were fully analyzable according to the FPRC reading protocol, thus prevalence of mtmDR was $23.8 \%$ (198/819). Of these, twenty-nine had CSDME according to fundus photography; 19 participants had centerinvolved DME according to OCT; and 42 participants had either CSDME and/or center-involved DME, with corresponding prevalences of $3.5 \%$ for CSDME, $2.3 \%$ for center-involved DME, and $5.1 \%$ for any DME according to both of these assessments. Average centerfield thickness was $239 \mu \mathrm{m}( \pm 0.05 \mu \mathrm{m})$ in the participants with CSDME (from fundus photographs only), and

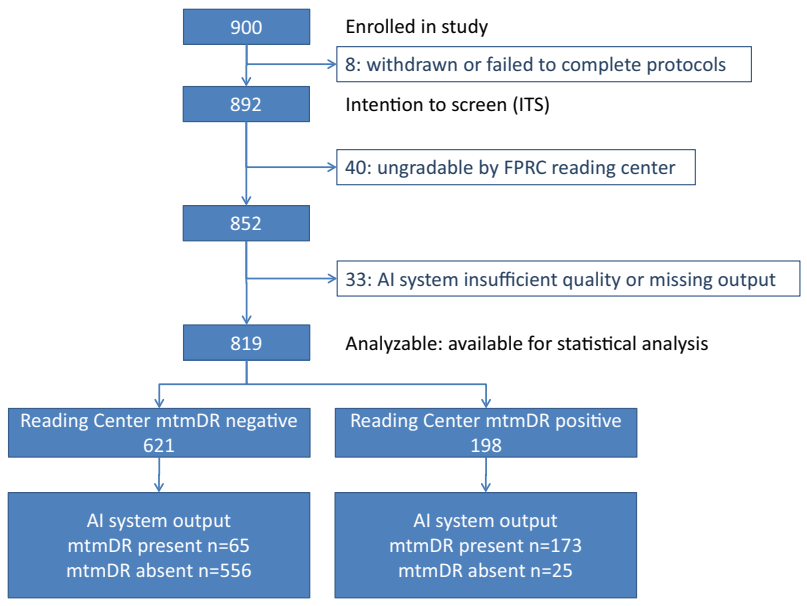

Fig. 1 Waterfall diagram showing the final disposition of each participant in the enrolled, intention to screen (ITS), and fully analyzable populations
$304 \mu \mathrm{m}( \pm 0.06 \mu \mathrm{m})$ in the participants with center-involved OCT DME. See Supplemental Table 1 for the DR frequency distribution according to ETDRS severity levels and to DME.

\section{Al system characteristics}

The Al system correctly identified 173 of the 198 fully analyzable participants with fundus $\mathrm{mtmDR}$. Logistic regression yielded a primary sensitivity of $87.2 \%(95 \% \mathrm{Cl}, 81.8 \%-91.2 \%)$ to fundus mtmDR, and $85.9 \%(95 \% \% \mathrm{Cl}, 82.5 \%-88.7 \%)$ to multimodal mtmDR. Observed sensitivity to fundus mtmDR was $87.4 \%$ (97.5\% Cl lower bound, 81.1\%) (173/198). The logistic regression model did not identify any significant effects for age, sex, race, ethnicity, $\mathrm{HbA1C}$, lens status, or site, on sensitivity. The retrospective power was $93 \%$. The Al system had a sensitivity to fundus vtDR of $97.4 \%$ (95\% Cl 86.2\%-99.9\%) (37/38), and to multimodal vtDR of $92.2 \%$ (95\% Cl $81.1 \%-97.8 \%)$ (47/51). Among these, it identified 28 of 29 (96.6\%; $95 \% \mathrm{Cl}, 82.2 \%-99.9 \%)$ participants with CSDME (fundus photographs only), 16 of 19 participants (84.2\%; $95 \% \mathrm{Cl}, 60.4 \%-96.6 \%)$ with center-involved DME (OCT only), and all participants with ETDRS level 43 or higher (including all 16 subjects with proliferative DR), with an $m t m D R$ detected output.

Among the 621 fully analyzable participants who did not have fundus mtmDR according to FPRC grading, there were 556 participants with an $m t m D R$ not detected output. Logistic regression yielded a primary specificity of $90.7 \%(95 \% \mathrm{Cl}$, $88.3 \%-92.7 \%)$ for fundus $\mathrm{mtmDR}$, and yielded $90.7 \%(95 \% \mathrm{Cl}$, $86.8 \%-93.5 \%)$ specificity for multimodal mtmDR, both after correction for enrichment. Observed specificity to fundus mtmDR was $556 / 621$ or $89.5 \%(97.5 \% \mathrm{Cl}$ lower bound, $86.5 \%)$. A logistic regression model did not identify any significant effects of sex, ethnicity, race, $\mathrm{HbA1C}$, lens status, or site, on specificity, while increased specificity was observed in subjects over 65 years of age $(p=0.030)$. The retrospective power was $87 \%$. See Table 2 .

Among the 73/892 non-analyzable participants, 40 (4\%) lacked a completed FPRC grading. In the worst case scenario (forcing all 40 subjects to a grading that is the opposite of the Al-system, in other words, the FPRC grading was set at mtmDR + if the Al system output was mtmDR not detected and vice versa), the sensitivity and specificity would have been $80.7 \%$ (two-sided $95 \%$ $\mathrm{Cl}, 76.7 \%-84.2 \%$ ) and $89.8 \%$ (two-sided 95\% Cl, 85.9\%-92.7\%) respectively. These results would still rule out the pre-specified inferiority hypotheses.

Of the 852 participants that received a completed FPRC grading, 33 participants (4\%) received an insufficient image quality output from the $\mathrm{Al}$ system after completion of the Al system protocol. Thus image-ability, defined as the percentage of participants with a completed FPRC grading and with a disease level Al system output, was $819 / 852$ (96.1\%; 95\% Cl, 94.6-97.28\%). In the 33 participants with $\mathrm{Al}$ system insufficient image quality, the prevalence of mtmDR was $10 / 33(30 \%)$, comparable to the mtmDR prevalence in the fully analyzable dataset. For the Al system protocol, $76.4 \%$ of participants did not require pharmacologic dilation, while $23.6 \%$ required dilation to obtain an Al system disease level output. The majority of participants, $64.7 \%$, completed the Al system protocol of 4 photographs the first time, $8.5 \%$ were able to complete the protocol after a single retry, $3.2 \%$ needed $2,19.7 \%$ needed $3,3.4 \%$ needed 4 and $0.5 \%$ needed five retries. There were $5 / 11$ subjects with enlarged optic disc cups, and 13/26 subjects with any drusen or RPE atrophy, received an " $m t m D R$ detected" output.

\section{DISCUSSION}

The results of this study show that the Al system in a primary care setting robustly exceeded the pre-specified primary endpoint goals with a sensitivity of $87.2 \%$ (>85\%), a specificity of $90.7 \%$ $(>82.5 \%)$, and an imageability rate of $96.1 \%$. Sensitivity is a patient 


\begin{tabular}{|c|c|c|c|}
\hline Age (years) & $<65$ & $69.1 \%(566 / 819)$ & $52.1 \%(38 / 73)$ \\
\hline \multirow[t]{2}{*}{ Ethnicity } & Hispanic or Latino & $16.4 \%(134 / 819)$ & $13.7 \%(10 / 73)$ \\
\hline & Not Hispanic or Latino & $83.0 \%(680 / 819)$ & $80.8 \%(59 / 73)$ \\
\hline \multirow{2}{*}{$\mathrm{HbA} 1 \mathrm{c} 7$} & $\geq 7$ & $86.7 \%(710 / 819)$ & $76.7 \%(56 / 73)$ \\
\hline & Unknown $^{*}$ & $1.7 \%(14 / 819)$ & $0 \%(0 / 73)$ \\
\hline \multirow[t]{3}{*}{$\mathrm{HbA1c9}$} & $<9$ & $46.4 \%(380 / 819)$ & $54.8 \%(40 / 73)$ \\
\hline & $\geq 9$ & $51.9 \%(425 / 819)$ & $45.2 \%(33 / 73)$ \\
\hline & Unknown ${ }^{*}$ & $1.7 \%(14 / 819)$ & $0 \%(0 / 73)$ \\
\hline \multirow{8}{*}{ Race } & Asian & $1.5 \%(12 / 819)$ & $4.1 \%(3 / 73)$ \\
\hline & Black & $28.2 \%(231 / 819)$ & $46.6 \%(34 / 73)$ \\
\hline & Mixed Race & $1.2 \%(10 / 819)$ & $1.4 \%(1 / 73)$ \\
\hline & Other-Mexican & $0.1 \%(1 / 819)$ & $0.0 \%(0 / 73)$ \\
\hline & Other-Puerto rican & $0.1 \%(1 / 819)$ & $0.0 \%(0 / 73)$ \\
\hline & Refuse to provide & $1.1 \%(9 / 819)$ & $0.0 \%(0 / 73)$ \\
\hline & Unknown & $3.5 \%(29 / 819)$ & $1.4 \%(1 / 73)$ \\
\hline & White & $63.9 \%(523 / 819)$ & $46.6 \%(34 / 73)$ \\
\hline
\end{tabular}

\begin{tabular}{|llll|}
\hline Table 2. & Al system diagnostic accuracy & \\
\hline & Point estimate & $95 \% \mathrm{Cl}$ & Superiority endpoint \\
\hline Sensitivity & $87.2 \%$ & $81.8 \%-91.2 \%$ & $85.0 \%$ \\
Specificity & $90.7 \%$ & $88.3 \%-92.7 \%$ & $82.5 \%$ \\
\hline
\end{tabular}

Point estimates for sensitivity and specificity were calculated on the 819 participants that were analyzable, using the prespecified logistic regression. The superiority endpoints were previously discussed with FDA.

safety criterion, because the Al system's primary role is to identify those people with diabetes who are likely to have DR that requires further evaluation by an eye care provider. Previous studies have shown that board-certified ophthalmologists that perform indirect ophthalmoscopy achieve an average sensitivity of $33 \%,{ }^{27} 34 \%$, or $73 \%{ }^{9}$ compared to the same ETDRS standard.

Specificity is also an important consideration, because it affects the number of people with diabetes who receive a referral but do not need one because they have only no or mild DR. Because all referrals will be evaluated by an eye care provider however, this will not increase the risk of that person receiving unnecessary treatment. The American Academy of Ophthalmology Preferred Practice Pattern (PPP 2017 revision) recommends that people with no or mild DR (ETDRS levels 10-20 and no DME) are followed annually, those with moderate DR (ETDRS level 35-47), and no DME receive more frequent follow-up, and those with more than moderate DR (level 53 or higher), or DME receive immediate evaluation. $^{26}$ The Al system had a sensitivity of $97.6 \%$ in identifying people that require immediate evaluation according to the PPP. Primary care providers may not feel comfortable evaluating the retina of a person with diabetes themselves. In that context, an autonomous-i.e., without human expert reading of the retinal images-Al system is helpful if it can identify those people who should receive referral to an eye care provider.

To our knowledge, the severity of DR and diabetic macular edema according to the ETDRS severity scale and OCT in a primary care diabetes population has not been determined previously. The only available studies reported on participants who were followed for some type of intervention, had some level of DR at inclusion, or did not have an ETDRS severity scale reading by a reading center. ${ }^{29-31}$ In the fully analyzable sample in this study, age, sex, ethnicity, and racial distribution were representative of the US diabetes population, ${ }^{7}$ and the prevalence of mtmDR in this representative sample was $23.8 \%$.

Additionally, the study yielded two reading center based gradings for DME: 1) CSDME, based on fundus photographs, and 2) center-involved DME, based on OCT. The prevalence of DME measured accordingly was lower than typically reported at $3.5 \%$ for CSDME, and $2.3 \%$ for center-involved DME. Previous studies reported that the majority of DME detected on OCT was detectable by fundus photography, but of the 19 cases in this study that had center-involved DME, only $6(32 \%)$ were identified as such by the FPRC reading center from fundus photographs. These results confirm an earlier report that fundus photography may be underestimating the prevalence and incidence of DME in people with diabetes, compared to OCT. ${ }^{32}$ Nevertheless, $84 \%$ of all cases with center-involved DME were detected by the Al system. Sensitivity and specificity met endpoints for fundus mtmDR, defined as ETDRS level $\geq 35$, or having CSDME, or both, all determined from fundus photographs only. It also met sensitivity and specificity endpoints for multimodal $m t m D R$, defined as ETDRS level $\geq 35$ (determined from fundus photographs), or having CSDME (determined from fundus photographs), or having centerinvolved DME (determined from OCT), or any combination of these. All cases of the most severe forms of DR, including proliferative DR, were detected. 
While there is widespread evidence for the effectiveness and cost-effectiveness of early detection of $\mathrm{DR}^{33}$ this is presently not the case for glaucoma, ${ }^{34}$ macular degeneration ${ }^{35}$ and many other eye diseases, and thus the present study was not designed or powered to analyze diagnostic accuracy on other retinal abnormalities in people with diabetes. However, we observe the following about so-called incidental findings: $6 / 819$ subjects with enlarged optic disc cups were not flagged by the Al system. Of these, an estimated $33 \%$ will have some form of glaucoma. ${ }^{36}$ Thus, $\sim 2 / 819$ subjects $(\sim 0.2 \%)$ would not have been referred to an eye care provider for disease while possibly having some form of glaucoma. Similarly, 13 subjects with drusen or RPE atrophy, all without retinal thickening, were not flagged, so $13 / 819$ subjects (1.6\%), would not have been referred to an eye care providersome of these with non-exudative age-related macular degeneration and intermediate drusen warranting preventative supplements. ${ }^{37}$ We wish to emphasize that these are observations only, given that the $95 \%$ confidence intervals around their estimates include $0 \%$.

As expected, sensitivity of the Al system was lower than that of the almost identical Al system tested on a laboratory dataset, which found a sensitivity of $97 \% .^{16}$ Sources for the lower sensitivity are likely to be:

- The use of stereo widefield photography, covering an area of the retina more than twice the size of that imaged for analysis by the Al system. Stereo photography allows CSDME to be estimated even in the absence of any other retinal lesions such as exudates. In the case of the laboratory study, the reference standard was determined from the same non-stereo images as available to the Al system, which does not allow the expert readers to estimate DME in the absence of exudates;

- The use of experienced ophthalmic photographers to obtain the reference standard stereo widefield photographs. This results in an overall higher image quality than that obtained by the primary clinic staff after $4 \mathrm{~h}$ of training that was available to the Al system;

- The prospective, ITS paradigm used in this study reduced selection and spectrum bias compared to laboratory studies.

The Al system is "physiologically plausible" to some degree, given its multiple, redundant, lesion-specific detectors for biomarkers, ${ }^{38}$ leading to increased robustness to small perturbations in input images, ${ }^{39}$ and because the biomarkers are based on over a century of worldwide clinical experience, lower expected risk of ethnic or racial bias. ${ }^{40}$ And in fact, diagnostic accuracy of the Al system was robust to sex, race, ethnicity, lens status and metabolic control, though specificity was higher in those over age 65 . This is likely related to the prevalence of highly reflective internal limiting membrane in younger people, which can be mistaken for exudates due to DME. ${ }^{12}$

As anticipated, the presence of lens opacities due to cataract significantly increased the number of imaging attempts required to get sufficient quality images, as well as the requirement for dilation, however sufficient image quality was obtained in $96.1 \%$. While selective dilation may be a challenge to scalable clinical implementation in some cases, the operator is explicitly advised on the need for dilation by the Al system: if an operator cannot capture three images of sufficient image quality without pharmacologic dilation, the system recommends the use of dilation drops.

In this regulated pivotal trial, the Al system was compared to the highest quality reference standard as determined by the FPRC, and met predetermined sensitivity and specificity standards for the autonomous detection of more than mild DR or DME in people with diabetes but no history of DR in primary care settings.

The results, in part, led FDA to authorize IDx-DR for "for use by health care providers to automatically detect more than mild diabetic retinopathy ( $\mathrm{mtmDR}$ ) in adults ( 22 years of age or older) diagnosed with diabetes who have not been previously diagnosed with DR", as, the first autonomous diagnostic Al system authorized by FDA in any field of medicine - without the need for a clinician to also interpret the image or results. ${ }^{41}$ At a high level, the results demonstrate the ability of autonomous Al systems to bring specialty-level diagnostics to a primary care setting, with the potential to increase access and lower cost. For people with diabetes, autonomous Al systems have the potential to improve earlier detection of DR, and thereby lessen the suffering caused by blindness and visual loss.

\section{METHODS}

\section{Autonomous Al diagnostic system}

The autonomous Al system, IDx-DR, has two core algorithms, an Image Quality Al-based algorithm, and the Diagnostic Algorithm proper. The complete Al system was locked before the start of this study (see below).

\section{Image quality algorithm}

The image quality algorithm is implemented as multiple independent detectors for retinal area validation as well as focus, color balance and exposure, and is used interactively by the operator to detect, in seconds, sufficient image quality for the Diagnostic algorithm to rule out (or in) $\mathrm{mtmDR}$, and thus maximize the number of subjects that can be imaged succesfully. As its input it takes four retinal images, and its output is whether quality is sufficient and if not, whether this is due to field of view or image quality. ${ }^{42}$

\section{Diagnostic algorithm}

The evolution of the diagnostic algorithm has been described extensively in publications spanning almost two decades..$^{12,18,19,43-45}$ It is a clinicallyinspired algorithm, and therefore has independent, validated detectors for the lesions characteristic for DR, including microaneurysms, hemorrhages and lipoprotein exudates, ${ }^{40}$ the outputs of which are then fused into a disease level output, using a separately trained and validated machine learning algorithm. ${ }^{46}$ The detectors have been implemented as multilayer convolutional neural networks (CNN), ${ }^{47}$ except the microaneurysm detector which is a multiscale featurebank detector, ${ }^{45,47}$ with substantially improved performance on a standardized laboratory dataset. ${ }^{16}$ In fact, in a laboratory study, its area under the receiver operator characteristics curve (AUC) of 0.980 (95\% Cl 0.968-0.992) was not statistically different from a perfect algorithm always outputting the truth, given the variability of the expert readers creating that truth. ${ }^{46}$

Each detector CNN was independently trained and validated to detect its assigned lesions from a region of a retinal image, using a total of over 1 million lesion patches from retinal images from people with and without DR. ${ }^{16,48}$ We consider these clinically inspired diagnostic algorithms with lesion-specific detectors for biomarkers, to be "physiologically plausible", as they mimic the functional organization human visual cortex. ${ }^{38}$ Such "physiologically plausible" systems with explicit, multiple, partially dependent detectors and a separate module for the higher level clinical decision have parallels in the human and primate ventral visual cortex, with specific subregions dedicated to the detection of particular categories of objects. $^{50-52}$ Downstream, in human experts, the higher level clinical decision is made in a part of the extrastriate cortex known as the fusiform face area, which is involved in making a clinical diagnosis from radiologic images, as has been found in functional imaging studies of radiologists when making clinical decisions. ${ }^{53}$

These physiologically plausible algorithms have been shown to be more robust to small perturbations in input images, possibly because they have partially dependent, and thus redundant detectors. ${ }^{39}$ Additionally, microaneurysms have been long recognized as the earliest retinal sign of DR that is seen on ophthalmoscopic examination, as recognized for the first time in the key paper by Friedenwald. ${ }^{40}$ However, decades before then, microaneurysms, and also hemorrhages, neovascularizations, IRMAs, exudates, and other abnormalities were already known to be the signs for $D R{ }^{54}$ Clinicians managing DR are aware that, although the incidence and prevalence of DR vary across racial, ethnic and age categories, the above signs are constant across races and ethnicities-in other words, whether or not someone with diabetes, showing multiple retinal hemorrhages and neovascularizations is of Hispanic or non-Hispanic descent, for instance, does not affect whether the clinician will diagnose 
Table 3. Study exclusion criteria

unable to understand the study

unable to or unwilling to sign the informed consent

indicate persistent vision loss, blurred vision, or floaters

previously diagnosed with macular edema, severe non-proliferative retinopathy, proliferative retinopathy, radiation retinopathy, or retinal vein occlusion

history of laser treatment of the retina or injections into either eye, or any history of retinal surgery;

currently participating in another investigational eye study or actively receiving investigational product for DR or DME

a condition that, in the opinion of the investigator, would preclude participation in the study;

contraindicated for imaging by fundus imaging systems used in the study because of hypersensitivity to light, recently underwent photodynamic

therapy, or was taking medication that causes photosensitivity

DR. Using detectors designed to detect these racially invariant biomarkers minimizes the risk of ethnic or racial bias in algorithm output.

The diagnostic algorithm uses four sufficient quality images and then takes seconds to make a clinical decision (at the point of care) and output a disease level indicating, whether more than mild DR and or macular edema is present.

Study design. From January 2017 to July 2017, 900 participants were prospectively enrolled in this observational study at 10 primary care practice sites throughout the United States. The study was approved by the institutional review board for each site, and all participants provided written informed consent. The study, which was funded by IDx LLC, was designed by the authors with input from the U.S. Food and Drug Administration (FDA) on the endpoints, statistical testing, and study design (see below). Emmes Corp, a contract research organization (CRO), provided overall project management, including data management and independent monitoring and auditing services for all sites. CCR, Inc., an Algorithm Integrity Provider (AIP), was contracted to lock the Al system, hold any intermediate and final results and images in escrow, and interdict access to these by the Sponsor, from prior to the start of the study until final data lock. Because the Sponsor was thus interdicted from access to the $\mathrm{Al}$ system, the AIP performed all necessary maintenance and servicing activities during the study as well as throughout closeout.

Study population. The target population was asymptomatic persons, ages of 22 and older, who had been diagnosed with diabetes and had not been previously diagnosed with DR. A diagnosis of diabetes was defined as meeting the criteria established by either the World Health Organization (WHO) or the American Diabetes Association (ADA); Hemoglobin A1c $(\mathrm{HbA} 1 \mathrm{c}) \geq 6.5 \%$ based on repeated assessments; Fasting Plasma Glucose $(\mathrm{FPG}) \geq 126 \mathrm{mg} / \mathrm{dL} \quad(7.0 \mathrm{mmol} / \mathrm{L})$ based on repeated assessments; Oral Glucose Tolerance Test (OGT) with two-hour plasma glucose (2-hr PG) $\geq$ $200 \mathrm{mg} / \mathrm{dL}(11.1 \mathrm{mmol} / \mathrm{L})$ using the equivalent of an oral $75 \mathrm{~g}$ anhydrous glucose dose dissolved in water; or symptoms of hyperglycemia or hyperglycemic crisis with a random plasma glucose (RPG) $\geq 200 \mathrm{mg} / \mathrm{dL}$ $(11.1 \mathrm{mmol} / \mathrm{L}){ }^{55,56}$ Exclusion criteria are listed in Table 3.

To help enroll a sufficient number of mtmDR participants for the evaluation of sensitivity, a stepwise enrichment strategy, as indicated in the prespecified protocol, was utilized mid-study to recruit sufficient numbers of $\mathrm{mtmDR}$ participants. The enrichment strategy sought higher risk participants with elevated $\mathrm{HbA} 1 \mathrm{c}(>9.0 \%)$ levels or elevated Fasting Plasma Glucose; this enrichment was independently activated by the statistician while always remaining masked to the Al system outputs and the ETDRS disease levels. To account for any unintentional spectrum bias in the no/mild population, the study pre-defined a specificity outcome parameter to correct for any potential spectrum bias resulting from this enrichment strategy as co-primary.

Site initiation. All primary care sites in the study identified one or more in house operator trainees to perform the Al system protocol (see below). After installation of the equipment by the Sponsor at the site, but before any participant was recruited, Al system operator trainees had to attest that they had not previously performed ocular imaging. Also, before start of study recruitment at each site, Al system operator trainees underwent a one-time standardized $4 \mathrm{~h}$ training program. They were trained how to acquire images, how to improve image quality if the $\mathrm{Al}$ system gave an insufficient quality output, and how to put images for analysis into the $\mathrm{Al}$ system. No additional training was provided to any of the Al system operators for the duration of the study. Independently, FPRC certified expert photographers were identified in geographic locations close to each site by the CRO, and documented 4W-D FPRC certification was required before any participant was imaged. ${ }^{22}$ The CRO independently completed site initiation visits at each site to ensure each site met all the good clinical practice requirements prior to start of enrollment.

Study protocol. All participants gave written informed consent to participate in both the Al system protocol, as well as the FPRC imaging protocol, using two different cameras:

The Al system protocol consisted of the following steps:

1. Operator takes images with a nonmydriatic retinal camera (NW400, Topcon Medical Systems, Oakland, NJ) according to a standardized imaging protocol with one disc and one fovea centered $45^{\circ}$ image per eye;

2. Operator submits images to the Al system for automated image quality and protocol adherence evaluation;

3. If the Al system outputs insufficient quality, steps 1-2 are repeated until sufficient quality is output or 3 attempts were made. If the Al system still indicates that images are of insufficient quality, the participant's pupils are dilated with tropicamide $1.0 \%$ eyedrops, (provided by the Sponsor at each site), until the pupil diameter is at least $5 \mathrm{~mm}$ in each eye or 30 minutes have passed, and steps $1-2$ are repeated until sufficient quality is output or 3 attempts were made. If the Al system still outputs that images are of insufficient quality, the Al system output of insufficient quality is automatically provided to the $C R O$ via secure data transfer;

4. Whenever the Al system indicates sufficient quality, the Al system disease level output (either $m t m D R$ detected or $m t m D R$ not detected) is automatically provided to the CRO via secure data transfer; the final Al system output provided to the CRO after this protocol was $m t m D R$ detected, mtmDR not detected or insufficient quality

The FPRC imaging protocol was then conducted, and consisted of the following steps, all performed by an FPRC certified photographer:

1. If participant is not already dilated, dilating eye drops of tropicamide $1.0 \%$ are administered;

2. Digital widefield stereoscopic fundus photography is performed, using a camera capable of widefield photography, (Maestro, Topcon Medical Systems, Oakland, NJ) according to the FPRC 4W-D stereo protocol, by an FPRC certified photographer; ${ }^{22}$

3. Anterior segment photography for media opacity assessment is performed according to the Age Related Eye Disease Study, ${ }^{57}$ by an FPRC certified photographer;

4. OCT of the macula is performed using a standard OCT system capable of producing a cube scan containing at least $121 \mathrm{~B}$ scans, (Maestro, Topcon Medical Systems, Oakland, NJ) according to the FPRC OCT protocol, by an FPRC certified photographer. ${ }^{22}$

The FPRC certified photographers were masked to the Al system outputs at all times.

Reference standards. The FPRC grading protocol consisted of determination of ETDRS Severity Scale (SS) levels for fundus photographs and standardized OCT grading, as follows: the 4W-D images were read by three experienced and validated readers at the FPRC according to the wellestablished ETDRS SS, using a majority voting paradigm. ${ }^{2,58}$ The macular OCT images were evaluated for the presence of center-involved DME by 
experienced readers at the FPRC according to the DRCR grading paradigm. ${ }^{24}$ For each participant, the ETDRS levels were mapped to $\mathrm{mtmDR}+$ (ETDRS level 35 or higher and /or DME present), or mtmDR(ETDRS level 10-20 and DME absent), taking the worst of two eyes to correspond to the outputs of the Al system at the participant level. ${ }^{16}$ To measure sensitivity for the cases requiring immediate followup, called vision threatening DR, we defined vtDR + as ETDRS level 53 or higher, and/ or DME present, See Supplemental Table 2 for the mapping from ETDRS and DME levels to dichotomous mtmDR- and $m \mathrm{tmDR}+$ and vtDR + . Because DME can be identified both on the basis of retinal thickening on stereoscopic fundus photographs, as well as on the basis of retinal thickening on OCT, we separately analyzed both. Stereoscopic fundusbased Clinically Significant DME (CSDME) was identified if there was either retinal thickening or adjacent hard exudates $<600 \mu \mathrm{m}$ from the foveal center, or a zone of retinal thickening $>1$ disc area, part of which is less than 1 disc diameter from the foveal center, according to the FPRC, in any eye. ${ }^{22,58,59}$ OCT based center-involved DME was identified if a participant had central subfield (a $1.0 \mathrm{~mm}$ circle centered on the fovea) thickness that was $>300 \mu \mathrm{m}$ according to the FPRC, in any eye. ${ }^{20}$ Accordingly, we further specify the definition of $\mathrm{mtmDR}$ where relevant:

\section{fundus $m t m D R+$ is defined as}

- ETDRS level $\geq 35$ (determined from fundus photographs)and/or

- CSDME (determined from fundus photographs)

and multimodal $m t m D R+$ is defined as:

- ETDRS level $\geq 35$ (determined from fundus photographs), and / or

- CSDME (determined from fundus photographs) and / or

- center-involved DME (determined from OCT).

and similarly for vtDR + . FPRC readers were masked to the Al system outputs at all times, masked to the fundus photograph reading when evaluating the OCT images, and masked to OCT readings when evaluating fundus photographs.

Primary and secondary outcomes. The primary outcomes were the sensitivity and specificity of the Al system, which had a pre-set threshold and was locked, to detect fundus-based $\mathrm{mtmDR}+$ according to the FPRC grading. The CRO received all final FPRC gradings and the final Al system outputs for all participants. FPRC staff, primary care site personnel, Sponsor personnel, and the statistical team were masked at all times to the Al system outputs. There were no interim analyses. The analysis was conducted following statistical analysis plan finalization and final database lock.

Statistical analysis. Study success was pre-defined as both sensitivity and specificity (see below) of the Al system in the US diabetes population. The hypotheses of interest were

$H_{0}: p<p_{0}$ vs. $H_{A}: p \geq p_{0}$

where $p$ is the sensitivity or specificity of the Al system and $p_{0}=75 \%$ for the sensitivity endpoint and $p_{0}=77.5 \%$ for the specificity endpoint under the null hypotheses. The alternative hypotheses were $85 \%$ for sensitivity and $82.5 \%$ for specificity, reflecting anticipated enrollment numbers, and pre-specified regulatory requirements. One-sided testing was further prespecified for both sensitivity and specificity; a one-sided $2.5 \%$ Type I error was used resulting in a one-sided $97.5 \%$ rejection rule per hypothesis. To preserve Type I error, study success was defined as requiring both null hypotheses to be rejected at the end of the study, e.g.

$P_{\pi}\left(H_{A} \mid\right.$ Data $)>0.975$.

The primary sensitivity calculation was performed using a logistic regression model including all $\mathrm{mtmDR}$ participants without any baseline covariate adjustment while the primary specificity calculation was performed using a logistic regression model with enrichment as a baseline covariate. A Firth adjustment was used to project sensitivity without any baseline covariate adjustment while the specificity was projected using absent enrichment status to diffuse spectrum bias ${ }^{60}$; enrichment was intended to increase the number of mtmDR cases based on stepwise increase of $\mathrm{HbA1C}$ levels, and thus expected to cause enrichment spectrum bias. Therefore, the specificity calculation was prespecified to correct for such spectrum bias; no such correction was prespecified for sensitivity analysis, because the goal was to shift the frequency of more severe DR cases. No data imputation was used for primary analyses.
Analyses were based on the data from the ITS population: participants who had valid results on both the FPRC imaging and reading protocol, and the Al system output, except where indicated; reported subgroup analyses were prespecified; subgroups $<10$ participants are not reported. Results are reported as posterior means, medians and with corresponding twosided $95 \%$ confidence intervals (CI). All analyses were conducted with the use of SAS software, version 9.1. Sample sizes for these hypotheses were calculated for at least $85 \%$ power and one-sided 2.5\% Type 1 error, requiring samples of $149 \mathrm{mtmDR}$ positive participants and $682 \mathrm{mtmDR}$ negative DR participants.

The study protocol and statistical analysis plan are available in the Supplementary information.

\section{Code availability}

The Al system described in this study is available as IDx-DR from IDx, LLC, Coralville, lowa. The underlying source codes are copyrighted by IDx, LLC, and are not available. No other custom code was used in the study.

\section{Data and materials availability}

The datasets generated during the current study that were used to calculate the primary outcome parameters are available upon reasonable request from the corresponding author, M.D.A., as well as from P.T.L.

\section{ACKNOWLEDGEMENTS}

The Wisconsin Fundus Photograph Reading Center (Barbara Blodi, MD, Amitha Domalpally MD and Dawn Myers) and Emmes Corp (Anne Hoehn, Project Manager) provided invaluable contributions to the study protocol. The study was registered before study start at ClinicalTrials.gov under NCT02963441.

\section{AUTHOR CONTRIBUTIONS}

P.T.L., M.D.A., J.C.F. designed the study; N.S. and M.B. acquired data; P.T.L. analyzed the data; all authors substantially revised the work and approved the submitted version. M.D.A. wrote the first draft of the manuscript, incorporating the other authors' contributions; the second author, P.T.L., devised the prospective study and analysis plan, oversaw the database lock process, analyzed the data after database lock, and edited the manuscript. All co-authors vouch for the data and adherence to the study protocol, which is available at this journal's website. All authors prospectively signed confidentiality agreements with IDx, LLC.

\section{ADDITIONAL INFORMATION}

Supplementary information accompanies the paper on the npj Digital Medicine website (https://doi.org/10.1038/s41746-018-0040-6).

Competing interests: M.D.A. is shareholder, director, and employee of IDx, LLC, and has relevant patents and patent applications assigned to the University of lowa; P.T.L. received fees from IDx, LLC for statistical consultancy; N.S. and M.B. declare no competing interests. J.C.F. is shareholder of IDx, LLC. Disclosure forms provided by the authors are available with the full text of this article. Patents (all issued) that may be affected by this study are: applied for by the University of lowa, inventor M.D.A., $7,474,775$, Automatic Detection of Red Lesions in Digital Color Fundus Photographs; 7,712,898, Methods and Systems for Optic Nerve Head Segmentation; 8,340,437, Methods and Systems for Determining Optimal Features for Classifying Patterns or Objects in Images; 9,924,867, Automated Determination of Arteriovenous Ratio in Images of Blood Vessels; applied by IDx, inventor M.D.A., 9,155,465, Snapshot Spectral Domain Optical Coherence Tomographer; 9,782,065, Parallel optical coherence tomography apparatuses, systems and related methods; 9,814,386, Systems and methods for alignment of the eye for ocular imaging.

Publisher's note: Springer Nature remains neutral with regard to jurisdictional claims in published maps and institutional affiliations.

\section{REFERENCES}

1. Hendricks, L. E. \& Hendricks, R. T. Greatest fears of type 1 and type 2 patients about having diabetes: implications for diabetes educators. Diabetes Educ. 24, 168-173 (1998).

2. Fong, D. S. et al. Diabetic retinopathy. Diabetes Care 26, 226-229 (2003).

3. Centers for Disease Control and Prevention. Diabetes Report Card 2012. (U.S. Department of Health and Human Services, Atlanta, GA, 2012). 
4. Bragge, P., Gruen, R. L., Chau, M., Forbes, A. \& Taylor, H. R. Screening for Presence or Absence of Diabetic Retinopathy: A Meta-analysis. Arch Ophthalm 129, 435-444 (2011).

5. National Health Service Diabetic Retinopathy Programme Annual Report, April 2007-March 2008 (2008).

6. Liew, G., Michaelides, M. \& Bunce, C. A comparison of the causes of blindness certifications in Englandand Wales in working age adults (16-64 years), 1999-2000 with 2009-2010. BMJ Open 4, e004015, https://doi.org/10.1136/ bmjopen-2013-004015 (2014).

7. Centers for Disease Control and Prevention. Centers for Disease Control and Prevention. National diabetes fact sheet: national estimates and general information on diabetes and prediabetes in the United States. (U.S. Department of Health and Human Services, Centers for Disease Control and Prevention, Atlanta, GA, 2008).

8. Hazin, R., Colyer, M., Lum, F. \& Barazi, M. K. Revisiting diabetes 2000: challenges in establishing nationwide diabetic retinopathy prevention programs. Am. J. Ophthalmol. 152, 723-729 (2011).

9. Lawrence, M. G. The accuracy of digital-video retinal imaging to screen for diabetic retinopathy: an analysis of two digital-video retinal imaging systems using standard stereoscopic seven-field photography and dilated clinical examination as reference standards. Trans. Am. Ophthalmol. Soc. 102, 321-340 (2004).

10. Abràmoff, M. D. \& Suttorp-Schulten, M. S. Web-based screening for diabetic retinopathy in a primary care population: the EyeCheck project. Telemed. J. E. Health 11, 668-674 (2005).

11. Scanlon, P. H. The English national screening programme for sight-threatening diabetic retinopathy. J. Med. Screen. 15, 1-4 (2008).

12. Abràmoff, M. D. et al. Automated analysis of retinal images for detection of referable diabetic retinopathy. JAMA Ophthalmol. 131, 351-357 (2013).

13. Abràmoff, M. D. et al. Automated early detection of diabetic retinopathy. Ophthalmology 117, 1147-1154 (2010).

14. Figueiredo, I. N., Kumar, S., Oliveira, C. M., Ramos, J. D. \& Engquist, B. Automated lesion detectors in retinal fundus images. Comput. Biol. Med. 66, 47-65 (2015).

15. Oliveira, C. M., Cristovao, L. M., Ribeiro, M. L. \& Abreu, J. R. Improved automated screening of diabetic retinopathy. Ophthalmologica 226, 191-197 (2011).

16. Abràmoff, M. D. et al. Improved automated detection of diabetic retinopathy on a publicly available dataset through integration of deep learning. Invest. Ophthalmol. Vis. Sci. 57, 5200-5206 (2016).

17. Gulshan, V. et al. Development and validation of a deep learning algorithm for detection of diabetic retinopathy in retinal fundus photographs. JAMA 316 2402-2410 (2016).

18. Abràmoff, M. D. et al. Evaluation of a system for automatic detection of diabetic retinopathy from color fundus photographs in a large population of patients with diabetes. Diabetes Care 31, 193-198 (2008).

19. Hansen, M. B. et al. Results of automated retinal image analysis for detection of diabetic retinopathy from the Nakuru Study, Kenya. PLoS ONE 10, e0139148 (2015).

20. Diabetic Retinopathy Clinical Research Network et al. Three-year follow-up of a randomized trial comparing focal/grid photocoagulation and intravitreal triamcinolone for diabetic macular edema. Arch. Ophthalmol. 127, 245-251 (2009).

21. PKC-DRS Group, et al. Effect of ruboxistaurin on visual loss in patients with diabetic retinopathy et al. Ophthalmology 113, 2221-2230 (2006).

22. Li, H. K. et al. Comparability of digital photography with the ETDRS film protocol for evaluation of diabetic retinopathy severity. Invest. Ophthalmol. Vis. Sci. 52, 4717-4725 (2011).

23. Gangaputra, S. et al. Comparison of standardized clinical classification with fundus photograph grading for the assessment of diabetic retinopathy and diabetic macular edema severity. Retina 33, 1393-1399 (2013).

24. Diabetic Retinopathy Clinical Research Network et al. Aflibercept, bevacizumab, or ranibizumab for diabetic macular edema. N. Engl. J. Med. 372, 1193-1203 (2015).

25. Wilkinson, C. P. et al. Proposed international clinical diabetic retinopathy and diabetic macular edema disease severity scales. Ophthalmology 110, 1677-1682 (2003).

26. American Academy of Ophthalmology Retina/Vitreous Panel \& Hoskins Center for Quality Eye Care. Diabetic Retinopathy PPP - Updated 2017 (San Francisco, CA: American Academy of Ophthalmology, 2017).

27. Pugh, J. A. et al. Screening for diabetic retinopathy: The wide-angle retinal camera. Diabetes Care 16, 889-895 (1993).

28. Lin, D. Y., Blumenkranz, M. S., Brothers, R. J. \& Grosvenor, D. M. The sensitivity and specificity of single-field nonmydriatic monochromatic digital fundus photography with remote image interpretation for diabetic retinopathy screening: a comparison with ophthalmoscopy and standardized mydriatic color photography. Am. J. Ophthalmol. 134, 204-213 (2002).

29. Diabetes Prevention Program Research Group. Long-term effects of lifestyle intervention or metformin on diabetes development and microvascular complications over 15-year follow-up: the Diabetes Prevention Program Outcomes Study. Lancet Diabetes Endocrinol. 3, 866-875 (2015).

30. [No authors listed] The relationship of glycemic exposure (HbA1c) to the risk of development and progression of retinopathy in the diabetes control and complications trial. Diabetes 44, 968-983 (1995).

31. Ahmed, J. et al. The sensitivity and specificity of nonmydriatic digital stereoscopic retinal imaging in detecting diabetic retinopathy. Diabetes Care 29, 2205-2209 (2006).

32. Wang, Y. T., Tadarati, M., Wolfson, Y., Bressler, S. B. \& Bressler, N. M. Comparison of prevalence of diabetic macular edema based on monocular fundus photography vs optical coherence tomography. JAMA Ophthalmol. 134, 222-228 (2016).

33. Klonoff, D. C. \& Schwartz, D. M. An economic analysis of interventions for diabetes. Diabetes Care 23, 390-404 (2000).

34. Moyer, V. A. \& U.S. Preventive Services Task Force. Screening for glaucoma: U.S Preventive ServicesTask Force Recommendation Statement. Ann. Intern. Med. 159, 484-489 (2013).

35. Chou, R., Dana, T., Bougatsos, C., Grusing, S. \& Blazina, I. Screening for impaired visual acuity in older adults: updated evidence report and systematic review for the US Preventive Services Task Force. JAMA 315, 915-933 (2016).

36. Hollands, $\mathrm{H}$. et al. Do findings on routine examination identify patients at risk for primary open-angle glaucoma? The rational clinical examination systematic review. JAMA 309, 2035-2042 (2013).

37. Age-Related Eye Disease Study Research Group. A randomized, placebo-controlled, clinical trial of high-dose supplementation with vitamins $C$ and $E$, beta carotene, and zinc for age-related macular degeneration and vision loss: AREDS Report 8. Arch. Ophthalmol. 119, 1417-1436 (2001).

38. Abràmoff, M. D. et al. Automated segmentation of the optic disc from stereo color photographs using physiologically plausible features. Invest. Ophthalmol. Vis. Sci. 48, 1665-1673 (2007).

39. Shah, Abhay, et al. "Susceptibility to misdiagnosis of adversarial images by deep learning based retinal image analysis algorithms." In Biomedical Imaging (ISB 2018), 2018 IEEE 15th International Symposium on, pp. 1454-1457. IEEE, 2018. https://ieeexplore.ieee.org/abstract/document/8363846/.

40. Friedenwald, J. \& Day, R. The vascular lesions of diabetic retinopathy. Bull. Johns. Hopkins Hosp. 86, 253-254 (1950).

41. US Food and Drug Administration. FDA permits marketing of artificial intelligence-based device to detect certain diabetes-related eye problems. https://www.fda.gov/NewsEvents/Newsroom/PressAnnouncements/ucm604357. htm April 12, 2018 (Washington, DC, 2018).

42. Niemeijer, M., Abràmoff, M. D. \& van Ginneken, B. Image structure clustering for image quality verification of color retina images in diabetic retinopathy screening. Med. Image Anal. 10, 888-898 (2006).

43. Abràmoff, M. D., Staal, J., Suttorp, M. S. A., Polak, B. C. \& Viergever, M. A. Low level screening of exudates and hemorrhages in background diabetic retinopathy. Comp. Assi. Fun. Image Anal., 15 (2000).

44. Niemeijer, M., van Ginneken, B., Russell, S. R., Suttorp-Schulten, M. S. A. \& Abràmoff, M. D. Automated Detection and Differentiation of Drusen, Exudates, and Cotton-Wool Spots in Digital Color Fundus Photographs for Diabetic Retinopathy Diagnosis. Invest. Ophthalmol. Vis. Sci. 48, 2260-2267 (2007).

45. Niemeijer, M., van Ginneken, B., Staal, J., Suttorp-Schulten, M. S. \& Abràmoff, M. D. Automatic detection of red lesions in digital color fundus photographs. IEEE Trans. Med. Imaging 24, 584-592 (2005).

46. Niemeijer, M., Abràmoff, M. D. \& van Ginneken, B. Information fusion for diabetic retinopathy $C A D$ in digital color fundus photographs. IEEE Trans. Med. Imaging 28, 775-785 (2009)

47. Krizhevsky, A., Sutskever, I. \& Hinton, G. E. ImageNet Classification with Deep Convolutional Neural Networks; in Advances in neural information processing systems 1097-1105 (Neural Information Processing Systems Foundation, Inc., California, 2012).

48. Quellec, G., Russell, S. R. \& Abràmoff, M. D. Optimal filter framework for automated, instantaneous detection of lesions in retinal images. IEEE Trans. Med. Imaging 30, 523-533 (2011).

49. Quellec, G. \& Abràmoff, M. D. Estimating maximal measurable performance for automated decision systems from the characteristics of the reference standard. application to diabetic retinopathy screening. Conf. Proc. leee. Eng. Med. Biol. Soc. 2014, 154-157 (2014).

50. Gauthier, I., Anderson, A. W., Tarr, M. J., Skudlarski, P. \& Gore, J. C. Levels of categorization in visual recognition studied using functional magnetic resonance imaging. Curr. Biol. 7, 645-651 (1997).

51. Polk, T. A. \& Farah, M. J. The neural development and organization of letter recognition: evidence from functional neuroimaging, computational modeling, and behavioral studies. Proc. Natl Acad. Sci. USA 95, 847-852 (1998).

52. Farah, M. J. \& Aguirre, G. K. Imaging visual recognition: PET and fMRI studies of the functional anatomy of human visual recognition. Trends Cogn. Sci. 3, 179-186 (1999). 
53. Harley, E. M. et al. Engagement of fusiform cortex and disengagement of lateral occipital cortex in the acquisition of radiological expertise. Cereb. Cortex 19, 2746-2754 (2009).

54. Lynch, S. K. \& Abràmoff, M. D. Diabetic retinopathy is a neurodegenerative disorder. Vision Res 139, 101-107 (2017).

55. American Diabetes Association. Classification and diagnosis of diabetes. Diabetes Care 38 Suppl, S8-S16 (2015).

56. American Diabetes Association. Diagnosis and classification of diabetes mellitus. Diabetes Care 37 Suppl 1, S81-90 (2014).

57. Chew, E. Y. et al. Evaluation of the age-related eye disease study clinical lens grading system AREDS report No. 31. Ophthalmology 117, 2112-2119 e2113 (2010).

58. Early Treatment Diabetic Retinopathy Study Research Group. Fundus photographic risk factors forprogression of diabetic retinopathy. ETDRS report number 12. Ophthalmology 98, 823-833 (1991).

59. Li, H. K. et al. Monoscopic versus stereoscopic retinal photography for grading diabetic retinopathy severity. Invest. Ophthalmol. Vis. Sci. 51, 3184-3192 (2010).
60. Firth, D. Bias reduction of maximum-likelihood-estimates. Biometrika $\mathbf{8 0}, \mathbf{2 7 - 3 8}$ (1993).

cc) (i) Open Access This article is licensed under a Creative Commons Attribution 4.0 International License, which permits use, sharing, adaptation, distribution and reproduction in any medium or format, as long as you give appropriate credit to the original author(s) and the source, provide a link to the Creative Commons license, and indicate if changes were made. The images or other third party material in this article are included in the article's Creative Commons license, unless indicated otherwise in a credit line to the material. If material is not included in the article's Creative Commons license and your intended use is not permitted by statutory regulation or exceeds the permitted use, you will need to obtain permission directly from the copyright holder. To view a copy of this license, visit http://creativecommons. org/licenses/by/4.0/.

(c) The Author(s) 2018 